Research Article

\title{
A Fuzzy Logic and Multilevel Analysis-Based Evaluation Algorithm for Digital Teaching Quality in Colleges and Universities
}

\author{
Zhong Han \\ Weifang University of Science and Technology, Weifang, Shandong 262700, China \\ Correspondence should be addressed to Zhong Han; middlehan@wfust.edu.cn
}

Received 10 August 2021; Revised 18 September 2021; Accepted 28 September 2021; Published 15 November 2021

Academic Editor: Mian Ahmad Jan

Copyright (C) 2021 Zhong Han. This is an open access article distributed under the Creative Commons Attribution License, which permits unrestricted use, distribution, and reproduction in any medium, provided the original work is properly cited.

\begin{abstract}
To address the limitations of the university digital teaching quality assessment algorithms as well as the large evaluation mistakes in the existing algorithms, this paper presents a unique university digital teaching quality evaluation method based on multilevel analysis. First, the existing state of digital teaching quality evaluation in colleges and universities is studied to develop an evaluation index for digital teaching quality. Then, to identify and compute the weight of digital teaching quality indicators, an index weight evaluation matrix is built and the weight of digital teaching quality assessment indicators is plotted using a multilevel structure tree model. Then, from the top to the bottom of the tree, this paper computes the hierarchical ranking of assessment indicators. Additionally, this paper computes the membership degree of index evaluation, normalises the evaluation indicators, and completes the digital teaching quality assessment with the digital teaching confidence calculation. The experimental results demonstrate that the proposed method's digital teaching quality assessment index has a high degree of accuracy and low evaluation error.
\end{abstract}

\section{Introduction}

Teaching quality evaluation describes the educational evaluation, where teaching is used as the evaluation object. It is the cornerstone and core of the entire educational assessment. Specific mathematical objectives, instructional norms, and standards are used to assess teaching quality. These include systematic detection and assessment of teaching and learning, assessing the teaching effect and the degree of realization of teaching objectives and making corresponding value judgements to enhance the teaching process using scientific and realistic techniques [1]. Currently, most Chinese colleges and universities are equipped with the latest hardware and software [2]. In the teaching process, we may actively employ contemporary teaching facilities, which can considerably enhance teaching quality. Under the premise of the same basic mathematics reform, different teachers still have their own teaching styles. Therefore, teachers' classroom teaching style has always affected the quality of the overall teaching process. In order to distinguish the differences in classroom teaching, there are not good enough scientific methods to encourage the excellent and make joint policies for the backward. Therefore, when evaluating a teacher's performance, completing the class hour index and the homework specified by the school and the teaching quality and effect have become a mere soft index, which greatly frustrates teachers' enthusiasm. At the same time, the classroom is where students learn the most, and classroom teaching is critical to enhance overall teaching quality [3]. The goal of developing a fair digital teaching quality rating system is to accurately discern between teacher quality and the white body benefits of digital education. Higher education is a critical component of Chinese education. The quality of higher education has a direct influence on the quality of higher-level skills and the level of growth of the national economy. The continuous enrollment expansion has gradually revealed the contradictions in teaching and scientific research equipment, 
quality and quantity of teachers, infrastructure construction, and other aspects of ordinary colleges and universities. The improvement of the gross enrollment rate has brought about the quality of students, which poses a potential threat to the quality of higher education [4].

With the increasing popularity of higher education in China, digital teaching has aided in the transformation of the whole educational system. The quality supervision and evaluation mechanism of digital teaching are important measures to ensure teaching quality [5]. It is critical to develop a fair and scientific method for monitoring and evaluating the quality of digital instruction. As a result, researchers in this sector have conducted a significant amount of quality evaluation study and produced positive outcomes. The authors in [6] presented a method for evaluating multimedia education quality using grey correlation analysis and neural networks. This technique creates a multimedia quality evaluation method based on grey correlation analysis and neural networks to address several flaws in multimedia quality evaluation. This technique creates a multimedia quality evaluation method based on grey correlation analysis and neural networks to address several flaws in multimedia quality evaluation. They use grey correlation analysis to calculate the weight of the multimedia teaching quality assessment index and a neural network to construct the classifier of multimedia teaching quality grade. Finally, various multimedia teaching quality evaluation methodologies are used to conduct the simulation test. The findings reveal that the multimedia teaching quality of the suggested technique is greater than $95 \%$. In comparison, the accuracy rate of the comparison method's multimedia teaching quality rating is less than $95 \%$. Simultaneously, the modelling efficiency of multimedia teaching quality evaluation has improved dramatically. It introduces a novel study approach for assessing the quality of multimedia education; however, it takes into account less digital teaching quality index data and has certain drawbacks. Zhang et al. [7] developed a supplemental teaching quality evaluation approach based on active learning support vector machine (SVM). This study provides an assessment index system for classroom teaching quality, taking into consideration the existing situation in a number of ways. A model for measuring classroom teaching quality is created using the active learning support vector machine. Experiments are done on the collected data set pertaining to a university's teaching quality. The experimental results demonstrate that the proposed evaluation model outperforms existing evaluation models in terms of accuracy and efficiency, and that it may produce superior teaching quality assessment outcomes in colleges and universities. This approach is straightforward, and its productivity is high, but there are few signs for the one-sided study object. Li et al. [8] offered a data miningbased methodology for evaluating university teaching quality. To begin, the model examines and analyses the current literature on college teaching quality assessment and determines the elements that influence college teaching quality evaluation. The model then gathers information on the elements that influence college teaching quality, assigns a grade to college teaching quality based on expert judgement, and creates a learning sample for college teaching quality evaluation. Finally, a data mining-based technique is presented to train the learning samples to construct the university teaching quality rating model using the BP neural network. Specific examples are used to illustrate the benefits of the university teaching quality model. The findings show that data mining may be used to describe differences in university teaching quality grades and provide high-precision university teaching quality evaluation results. Furthermore, compared with previous methods, the college teaching quality evaluation error is far lower, which has significant benefits. However, the assessment of digital teaching quality is woefully inadequate and has to be improved.

In light of the shortcomings of the previous techniques, this work offers a study of a multilevel analysis-based algorithm for evaluating digital teaching quality in colleges and universities. The following are the precise technical procedures examined in this paper:

Step 1. I examine the present state of digital teaching quality assessment in colleges and universities and calculate the digital teaching quality evaluation index in colleges and universities based on the existing scenario.

Step 2. To establish the weight of digital teaching quality evaluation indicators, I create an index weight evaluation matrix.

Step 3. I design a multilevel structure tree model and then establish that the assessment indicators are ranked from top to bottom hierarchically. I also compute the evaluation indicators' membership degree, normalise the evaluated indicators, and finish the evaluation of digital teaching quality by calculating the confidence of digital teaching indicators.

\section{Evaluation Index and Weight of Digital Teaching Quality}

\subsection{Current Status of the Digital Teaching Quality Evaluation} in Universities. Personal experience, use of students' scores, teachers' self-evaluation, comprehensive scoring of leaders, and then seeking the average score, or subjectively scoring directly through some characteristics in the teaching process, are still used to evaluate digital teaching quality in colleges and universities (such as outstanding teaching contributions and teaching accidents). This means that the manual level is based on qualitative analysis. However, due to the large amount of data collected and statistically summarized, manual teaching quality evaluation methods often make information timeliness and accuracy challenging to guarantee. Such evaluation method still relies on personal experience and focuses on qualitative analysis, lacking objectivity, and quantitative analysis. Especially when making a comprehensive evaluation of teachers' long-term work, it is neither comprehensive nor in-process to evaluate a specific period or some prominent characteristics [9]. 
To begin with, several indicators are frequently used in the evaluation of digital teaching quality in colleges and universities, and the indicators used should properly represent the activity of teachers. We must not make a partial and comprehensive assessment based on the current state of one or more indicators. This assessment is unfair and unscientific since it only evaluates the remarkable contribution of teaching in some areas or only stresses rare teaching errors [10].

Second, the school assesses the quality of digital instruction in colleges and universities by rating students for teachers, combining the scores of leaders for teachers and then using statistics to arrive at an average score. This assessment technique appears to be "all-encompassing," yet it is not. The correlation and mutual relevance of numerous indicators are ignored in this technique. Because not every metric is equally important in the teaching process, and from an empirical standpoint, using manual techniques to determine the scores of digital teaching quality evaluation in colleges and universities is time-consuming and tedious. Still, it does not achieve the objective and fair evaluation of digital teaching quality.

Finally, the weighting mechanism and assessment index are unscientific. Two characteristics of an assessment system have the most influence on the evaluation object. On the one hand, whether the evaluator can objectively evaluate the research object; nevertheless, given the current technical conditions, the appraiser's ability to evaluate the evaluation object objectively is not guaranteed [11].

On the other side, it is to determine whether the index system and its weight are fair. In this regard, the index system and its weight of certain evaluation systems are introduced to the system during system construction based on user needs, which cannot be altered during user usage and lack flexibility.

2.2. Evaluation Index of Digital Quality Teaching. I applied the fuzzy comprehensive evaluation theory [12] to create a digital teaching quality assessment index based on the current state of digital quality evaluation. How to appropriately estimate each assessment index's weight is a crucial topic in both theory and practise of fuzzy comprehensive evaluation. It provides assessment results based on numerous criteria (or many judges) that evaluate something, and it combines the evaluation findings [10] of the various elements (or multiple judges).

Suppose $U$ and $L$ represent two limited theories of college digital quality evaluation, respectively,

$$
\begin{aligned}
U & =\left\{u_{1}, u_{2}, \cdots, u_{n}\right\}, \\
L & =\left\{l_{1}, l_{2}, \cdots, l_{n}\right\} .
\end{aligned}
$$

Among them, $U$ represents a collection of digital quality evaluation factors (or judges) and $L$ denotes the collection of college digital quality evaluation comments (or the collection of evaluation results). For the digital quality assessment list factors of universities $u_{i n}$, the fuzzy evaluation given for something uses a fuzzy set of digital quality evaluation of a university on the comment set (or evaluation result set) as follows:

$$
U_{m}=\left(u_{i 1}, u_{i 2}, \cdots, u_{i n}\right) .
$$

Among them, $0 \leq u_{i j} \leq 1$, where $i=1,2, \cdots, n$ and $j=1,2, \cdots, m$.

Then, the evaluation matrix of the digital quality evaluation index $P$ can be obtained as follows:

$$
P=\left[\begin{array}{cccc}
u_{11} & u_{12} & \cdots & u_{1 n} \\
u_{21} & u_{22} & \cdots & u_{2 n} \\
\vdots & \vdots & \ddots & \vdots \\
u_{n 1} & u_{n 2} & \cdots & u_{n n}
\end{array}\right]
$$

The evaluation index of university digital quality mainly adopts matrix method for evaluation, and at the same time, it must meet the conditions of formula 3 , in which $U$ and represents fuzzy sets $\left(x_{1} /\left(u_{1}, x_{2} / u_{2}, \cdots, x_{n} / u_{n}\right)\right)$ or a vector $X=\left(x_{1}, x_{2}, \cdots, x_{n}\right)$, and $\sum_{i=1}^{n} x_{i} \geq 0$ represents the comprehensive evaluation result of the indicator or a vector. It was obtained in accordance to the above calculation and determines of college digital teaching indicators [13].

\subsection{Weight Calculation of Digital Teaching Quality} Evaluation Index in Universities. After obtaining the digital teaching indicators from the aforementioned colleges, the weight [14] of the above assessment indicators must be calculated by creating an online teaching quality evaluation matrix. The evaluation matrix is expressed by $R$. The above teaching quality evaluation indicators have a subset $R_{i}$. In this paper, I set all teaching quality influence factors as $R$ and construct the evaluation matrix of $T \times \mathrm{V}$ in the feasible field of influence factors, represented by $R$,

$$
R=\left[\begin{array}{cccc}
r_{11} & r_{12} & \cdots & r_{1 n} \\
r_{21} & r_{22} & \cdots & r_{2 n} \\
\vdots & \vdots & \cdots & \vdots \\
r_{m 1} & r_{m 2} & \cdots & r_{m n}
\end{array}\right]
$$

Through the evaluation of digital teaching quality, the calculation process is as follows:

$$
S^{\prime}=Q \cdot R=\left(q_{1}, q_{2}, \ldots, q_{m}\right)\left[\begin{array}{cccc}
r_{11} & r_{12} & \cdots & r_{1 n} \\
r_{21} & r_{22} & \cdots & r_{2 n} \\
\vdots & \vdots & \cdots & \vdots \\
r_{m 1} & r_{m 2} & \cdots & r_{m n}
\end{array}\right]=\left(s_{1}^{\prime}, s_{2}^{\prime}, \ldots, s_{n}^{\prime}\right) \text {. }
$$

In order to improve the accuracy of the impact of this research on the digital teaching quality, the membership and evaluation membership value are repeatedly calculated, and the evaluation value of all the influence indicators of the digital teaching quality is obtained, which is denoted by SS. Quantitative fuzzy comprehensive evaluation results of online classroom teaching quality can be calculated as follows: 


$$
S \times N=\left(s_{1}, s_{2}, \ldots, s_{n}\right)\left[\begin{array}{c}
n_{1} \\
n_{2} \\
\vdots \\
n_{n}
\end{array}\right] .
$$

In the weight determination and calculation of digital teaching quality indicators in universities, the framework index weight evaluation matrix determines the weight of digital teaching quality evaluation indicators, which lays the foundation for the subsequent evaluation.

\section{Implementation of College Digital Teaching Quality Evaluation Algorithm Based on Multilevel Analysis}

Multilevel analysis is a frequently used and essential approach in fuzzy mathematics. It consists of a number of assessment indicators as well as the weights assigned to each indication across all indicators. The term "multilevel" refers to the hierarchy of indicators rather than the assessment indicators themselves. In the form of a tree structure, these indicators are interconnected [15]. The fundamental structure of these indicators is depicted in Figure 1.

A complicated problem is divided into numerous components using this structural paradigm (or elements). Each component can also be broken down into multiple groups, each with its own hierarchy. These levels are divided into three groups in this study:

(a) Target Layer. It is the assessment objective of the evaluation object and reflects the system evaluation objective or the predefined aim or outcome of the analytical problem. Only one target or element may be found at the highest level at this level.

(b) Criteria Layer. It depicts the goal's intermediary connection, such as the plan, measurements, and tactics used to attain it. It is a set of indications or rules for evaluating anything. The criteria layer, the subcriterion layer, each subcriterion, and the link between the criterion layer are all possible levels. The subcriterion layer depicts the relationship between the preceding and subsequent layers. Each level can have many criteria or components, with sublevels reporting to the preceding level's criterion.

(c) Index Layer. This layer shows the objective's intermediate connection, as well as the solutions, measurements, methods, and other options for accomplishing the goal. You can have many schemes or elements at this level, which is the lowest level.

In the digital teaching quality evaluation, after determining the digital teaching indicators and weights, the multilevel analysis method is used to judge the key digital teaching indicators. I set the importance indicator data in the index collection as $B_{i j}$ and then carry on it the judgement $n(n-1) / 2$. I define the membership of the multilevel evaluation index as follows:

$$
b_{i}=\sum_{i=1}^{m} \omega_{i} \times s_{i j}=\min \left\{1, \sum_{i=1}^{m} \omega_{i} \times s_{i j}\right\} .
$$

In formula, $i=1,2, \cdots, m$ and $j=1,2, \cdots, n$ indicate a fuzzy addition, $i$ represents the $i$ evaluation factor of the $n$ evaluation factors, $j$ represents the $j$ rating of the $m$ evaluation rating, and $b$ represents the membership of the $j$ evaluation rating.

According to [16], the multilevel analysis is evaluated by determining the hierarchy of the evaluation indicators. Identifying the hierarchical ranking of evaluation metrics is performed in a high-end order. The multilevel structure of digital teaching quality evaluation is shown in Figure 2:

In Figure 2, there are $m$ indicators in layer $K$, and the weight of the total digital teaching quality evaluation is $k_{1}, k_{2}$, and $k_{3}$. At this time, the $K$ layer contains $n$ indicators $(\underline{k}-1)$, sorted in this order. If the index $\mathrm{k}-1$ is independent of the index $(k-1)_{j}$, the value is 0 ; then, the evaluation result is as follows:

$$
w_{i}^{(k-1)}=\sum_{j=1}^{m} w_{j}^{(k)} w_{i j}
$$

With the digital teaching quality evaluation error, further processing is first normalized as follows:

$$
w_{i}=\frac{\overline{w_{i}}}{\sum \overline{w_{j}}} .
$$

The confidence of the normalized evaluation results is calculated to determine that whether or not the final evaluation results are credible as follows:

$$
\vartheta^{2}=\frac{\sum\left(b_{i j}^{k}-\overline{b_{i j}^{k 2}}\right)}{m} \text {. }
$$

In the formula, $\vartheta^{2}$ represents the positive distribution, $b_{i j}^{k}$ is the overall variance, and $b_{i j}^{k 2}$ represents the overall standard deviation.

In the digital teaching quality evaluation, I first design the multilevel structure tree model and then determine the hierarchy of the evaluation indicators from the order of the evaluation. Further, I calculate the membership of the evaluation indicators through the confidence of digital teaching indicators and finally compute the evaluation of digital teaching quality evaluation.

\section{Experimental Analysis}

4.1. Experimental Protocol Setup. An experimental study is carried out in this work to validate the performance of the suggested assessment technique. The experiment used English majors at a university for a year as the research subject. The class had a total of 80 pupils. Multimedia equipment was used to teach the English majors in the class. In a week, multimedia instructors taught three professional classes. The grade served as the control group. The teaching of professional courses in this class is not done in multimedia for students in class B. The efficacy 


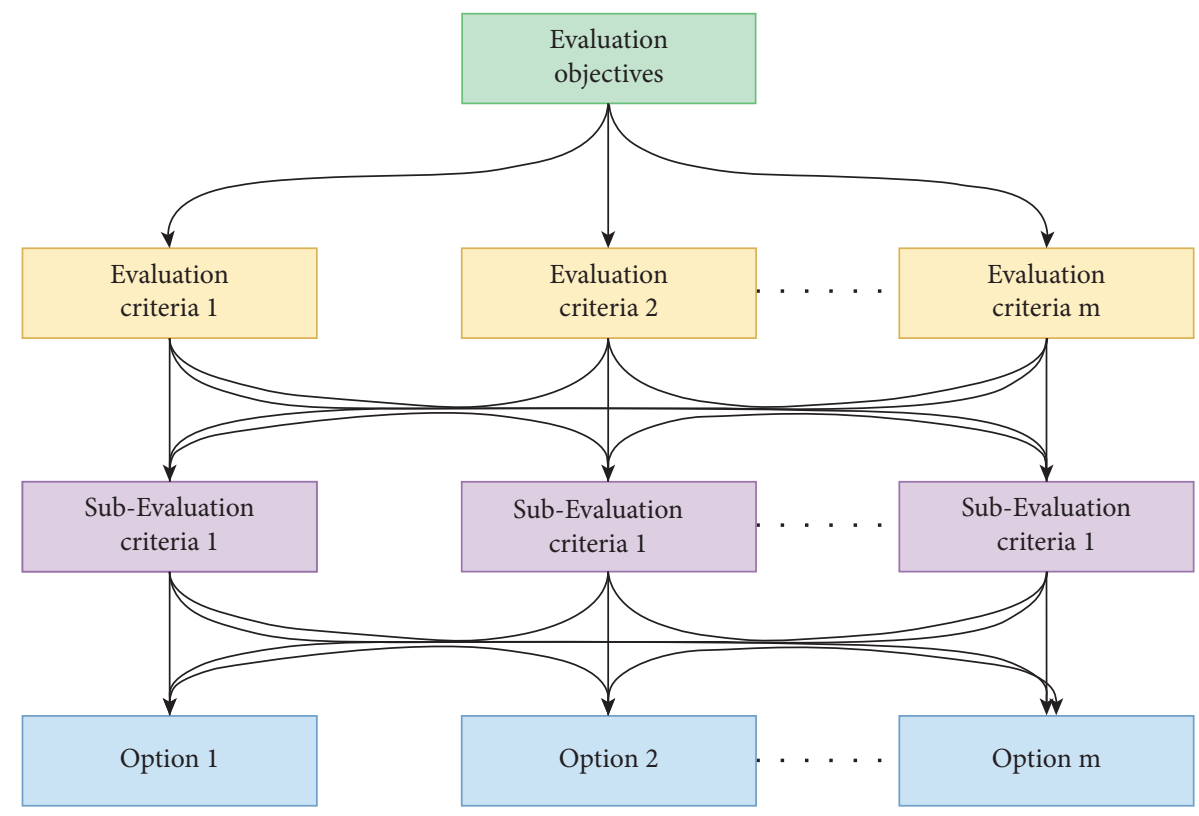

Figure 1: Tree structure diagram of multilevel analysis.

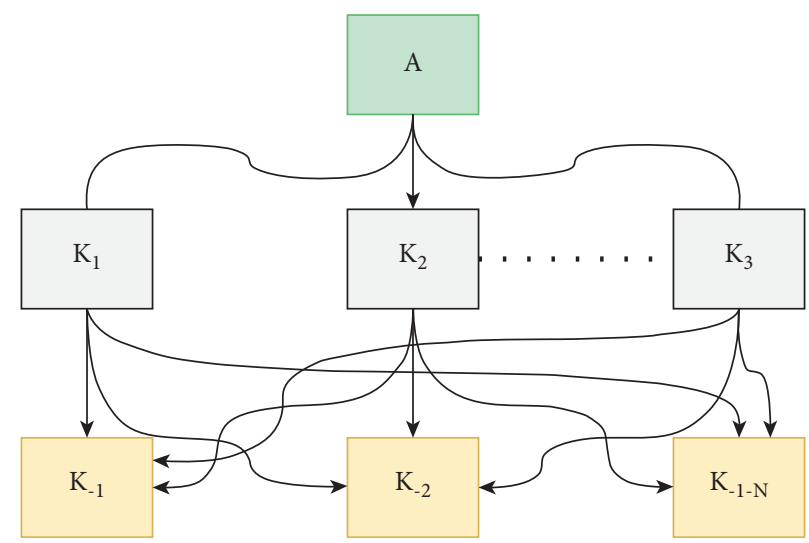

FIgURE 2: The multilevel structure of digital teaching quality evaluation.

of the evaluation technique is proven by assessing the academic accomplishments of classes $\mathrm{A}$ and $\mathrm{B}$ over the course of one semester. An experimental study is carried out in this work to validate the performance of the suggested assessment technique. The experiment used English majors at a university for a year as the research subject. The data collected in the experiment are processed by professional software to verify the experiment's correctness.

4.2. Experimental Index Design. The experiment's performance analysis is influenced by the indicators used in the experiment. As a result of the comparison technique, the accuracy of evaluation indicators and the error of quality assessment are used as experimental indicators in this paper's experimental analysis. The experiment's middle school kids' accomplishment data have been provided, and the experimental analysis is based on the students' successes.
4.3. Analysis of Experimental Results. The proposed approach, multimedia teaching quality evaluation based on grey correlation analysis and neural network, and the correctness of the data mining algorithm were used to test the method's performance. Table 1 summarises the findings.

Table 1 and Figure 2 illustrate the accuracy results of the suggested technique based on grey correlation analysis and the data mining algorithm-based college teaching quality rating model. The accuracy in the sample evaluation data is approximately 96 percent, the accuracy based on grey correlation analysis and multimedia teaching quality evaluation is approximately 89 percent, and the college teaching quality evaluation model based on the sample evaluation data is approximately 85 percent. The determined indications of this approach, on the other hand, are more accurate. This is due to the method's usage of fuzzy theory, which increases our method's efficacy. Precision comparison results through different method evaluation indexes (\%) are shown in Figure 3. 
TABLe 1: Analysis of precision comparison results determined by different method evaluation indexes (\%).

\begin{tabular}{lccc}
\hline $\begin{array}{l}\text { Number of index } \\
\text { determination/times }\end{array}$ & $\begin{array}{c}\text { The proposed } \\
\text { method }\end{array}$ & $\begin{array}{c}\text { Multimedia teaching quality } \\
\text { evaluation based on grey association analysis and } \\
\text { neural network }\end{array}$ & $\begin{array}{c}\text { Evaluation of college teaching } \\
\text { quality based on the data mining } \\
\text { algorithm }\end{array}$ \\
\hline 10 & 95 & 89 & 85 \\
20 & 96 & 88 & 85 \\
30 & 95 & 88 & 84 \\
40 & 95 & 85 & 86 \\
50 & 95 & 85 & 85 \\
60 & 96 & 84 & 84 \\
70 & 96 & 86 & 80 \\
80 & 95 & 85 & 82 \\
90 & 96 & 84 & 81 \\
100 & 95 & & 81 \\
\hline
\end{tabular}

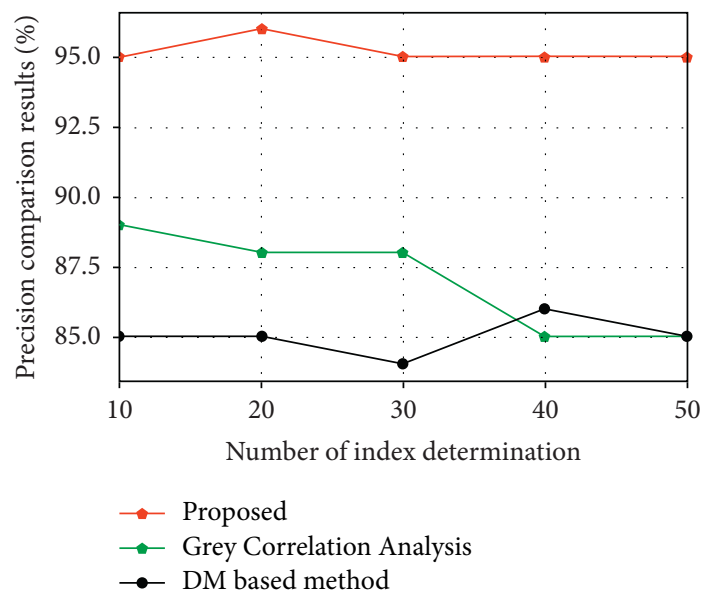

FIgURE 3: Precision comparison results through different method evaluation indexes (\%).

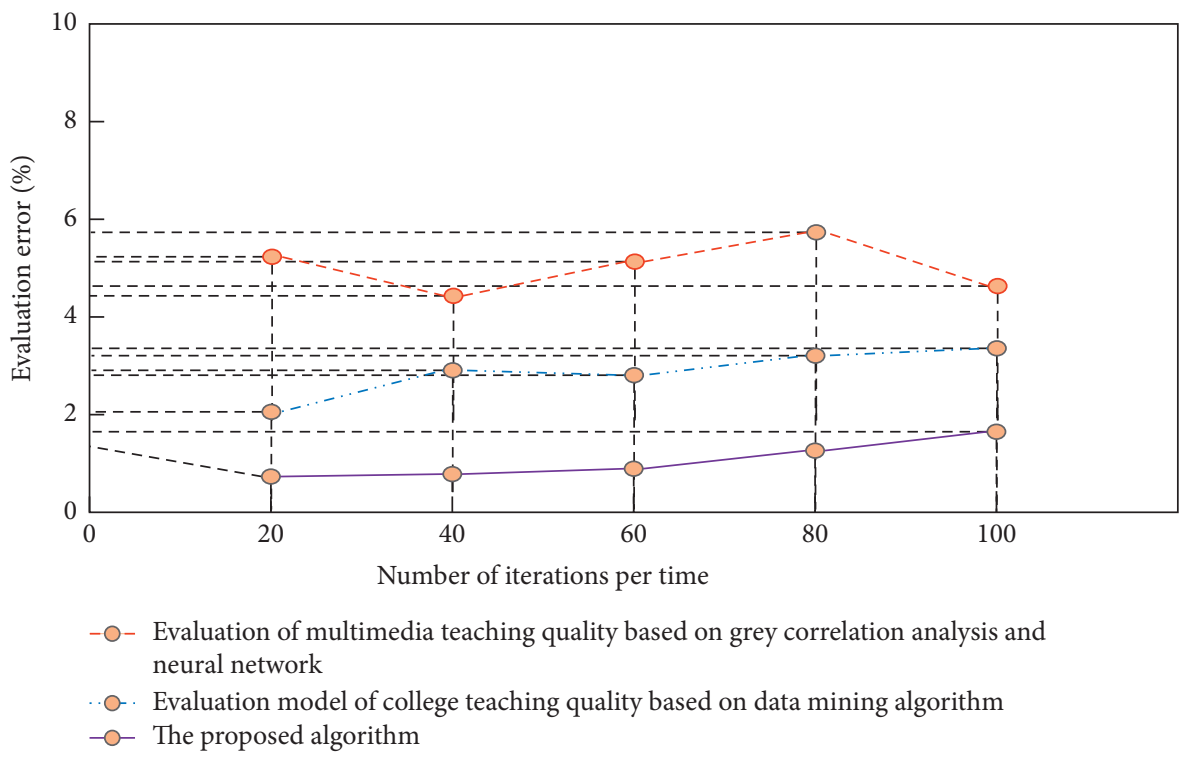

FIGURE 4: Error results of the sample evaluation data evaluation.

The proposed model, grey correlation analysis, neural network multimedia teaching quality assessment, and college teaching quality evaluation model were used to evaluate the sample evaluation data evaluation error. Figure 4 depicts the results of the sample evaluation data evaluation error.
Figure 4 shows that the proposed approach, grey correlation analysis and neural network multimedia teaching quality assessment, and the college teaching quality evaluation model based on data mining algorithm are distinct. The suggested approach has the lowest sample evaluation 
TABLE 2: Comparison results with respect to consistency examination $(\mathrm{CR})$ values.

\begin{tabular}{lccc}
\hline Methods & CI & RI & CR \\
\hline Proposed method & 0.00843 & 0.845 & 0.01 \\
Neural network & 0.4528 & 0.645 & 0.67 \\
Data mining & 0.5142 & 0.598 & 0.84 \\
\hline
\end{tabular}

error, which is always less than $2 \%$, while the other two methods are always greater. This is because the proposed approach calculates the membership of the index evaluation, completes the evaluation of the quality of digital teaching indicators, and then enhances the proposed method's efficacy.

Table 2 reveals that only the consistency examination (CR) score of this approach is less than 0.1 among the three methods. In the university teaching quality evaluation model based on data mining algorithms, CR values are all above 0.1 . This indicates that the technique and the actual evaluation findings are the most consistent, indicating that the method is successful.

\section{Conclusion}

This paper proposes a multilevel analysis-based digital teaching quality evaluation model. This paper created a hierarchical tree-based model based on the digital teaching quality index and the weight of the assessment matrix. In my proposed method, I first determined the evaluation's membership and then normalised the digital teaching index confidence before concluding the digital teaching quality assessment. The experimental findings demonstrate that the digital teaching quality evaluation indexes are very accurate while being difficult to implement [16].

\section{Data Availability}

The data used to support the findings of this study are available from the corresponding author upon request.

\section{Conflicts of Interest}

The author declares that there are no conflicts of interest or personal relationships that could have appeared to influence the work reported in this paper.

\section{References}

[1] Y. Guang-Hua and S.-Y. Wang, "Research on university evaluation of teaching quality based on data driven," in Proceedings of the 2019 3rd International Workshop on Education, Big Data and Information Technology, Istanbul, Turkey, October 2019.

[2] M. Matore, N. Othman, and E. Matore, "The associations of peer review and self-reflections in teaching assessment with accuracy and gender from TVET teachers' feedback," in Proceedings of the Mechanical Engineering Research Day, Malaysia, March 2020.

[3] J. Kriewaldt, R. Walker, V. Morey, and M. Chad, "Activating and reinforcing graduates' capabilities: early lessons learned from a teaching performance assessment," Australian Educational Researcher, vol. 48, no. 1, pp. 1-16, 2021.

[4] H. Wu, "Multimedia interaction-based computer-aided translation technology in applied english teaching," Mobile Information Systems, vol. 2021, Article ID 5578476, 2021.

[5] R. S. Crane and W. Kuyken, "The mindfulness-based interventions: teaching assessment criteria (MBI:TAC): reflections on implementation and development," Current Opinion in Psychology, vol. 28, pp. 6-10, 2019.

[6] H. Du, "An English network teaching method supported by artificial intelligence technology and WBIETS system," Scientific Programming, vol. 2021, Article ID 8783899, 2021.

[7] Y. Zhang, "Assisted teaching quality evaluation model based on active learning support vector machine," Modern Electronics Technique, vol. 42, no. 7, pp. 112-114, 2019.

[8] Y. Li, "Design of university teaching quality evaluation model based on data mining algorithm," Modern Electronics Technique, vol. 43, no. 17, pp. 119-122, 2020.

[9] L. F. Education, "An assessment of history teaching strategies and promotion of professional diversity in public high schools in mezam, Cameroon," Quarterly Review, vol. 3, 2020.

[10] H. Lee, "What are the effects of the change in the assessment systems on the alignment between curriculum, teaching and assessment? a case in Korean middle schools," Journal of Asia TEFL, vol. 16, no. 14, pp. 45-52, 2019.

[11] Y. Yang, "Quality evaluation method of a mathematics teaching model reform based on an improved genetic algorithm," Scientific Programming, vol. 2021, Article ID 6395349, 2021.

[12] M. W. Zackoff, F. J. Real, D. Deblasio et al., "Objective assessment of resident teaching competency through a longitudinal, clinically integrated, resident-as-teacher curriculum," Academic Pediatrics, vol. 19, no. 6, pp. 698-702, 2019.

[13] X. Wei, "Quality-centered mobile terminal teaching assessment system under the background of big data," in Proceedings of the 2020 5th International Conference on Smart Grid and Electrical Automation (ICSGEA), IEEE, Zhangjiajie, China, 2020.

[14] Z. Wang, "Interpreting common European framework of reference for languages: learning, teaching, assessment, companion volume with new descriptors," Foreign Language Testing and Teaching, vol. 12, no. 2, pp. 1145-1148, 2019.

[15] K. Haghdar, "Optimal DC source influence on selective harmonic elimination in multilevel inverters using teachinglearning based optimization," IEEE Transactions on Industrial Electronics, vol. 9, no. 99, p. 1, 2019.

[16] X. Zhang, "Planning the structure of university teaching staff based on multiobjective optimization method," Scientific Programming, vol. 2021, Article ID 1773561, 2021. 